\title{
Hydroxyapatite Deposition Disease- Spontaneous Resolution of Significant Post Treatment Deposit
}

\section{Agarwal S}

Pandit Bhagwat Dayal Sharma, Post Graduate, Institute of Medical Sciences, Rohtak, India

${ }^{*}$ Corresponding author: Agarwal S, Pandit Bhagwat Dayal Sharma, Post Graduate, Institute of Medical Sciences, Rohtak, India, Tel:+91-9355622099, E-mail: agarwalsdr@gmail.com

Citation: Agarwal S (2019) Hydroxyapatite Deposition Disease- Spontaneous Resolution of Significant Post Treatment Deposit. J Case Rep Stud 7(1): 101. doi: 10.15744/2348-9820.7.101

Received Date: September 20, 2018 Accepted Date: February 25, 2019 Published Date: February 27, 2019

\begin{abstract}
Calcific deposits are commonly seen on imaging done for other reasons. However, it can also present with periarthritis and adhesive capsulitis leading to severe disabling pain. Many theories have been proposed to explain the etiology and it is considered idiopathic by some. The management is also debatable, Barbotage being the most popular. In the majority of the cases it disappears spontaneously, requiring intervention like Barbotage and surgery only in a few cases. We present a case of a 45 year old female patient who had these calcific deposits in an unusual location of her right shoulder. These disappeared partially on conservative treatment. Subsequently, she developed severe pain in her right shoulder with restriction of all her movements. The pain was severe enough to interfere with her sleep. As a result she underwent barbotage. During the procedure an attempt was made to aspirate the calcific deposit in its entirety. This was not possible due to clogging of needle several times. There was complete resolution of pain following the procedure. However, post- procedure Magnetic resonance imaging (MRI) revealed a significant residual calcification which completely resolved 02 months after therapy with no recurrence.
\end{abstract}

Keywords: HADD; Shoulder; Barbotage

\section{Introduction}

Hydroxyapatite deposition disease (HADD) is a common disease with calcific deposits occurring in para-articular or intra-articular location and can involve varied anatomical sites [1]. It usually affects women in 40-60 years of age. Severe disabling pain leading to periarthritis and adhesive capsulitis can occur in few cases [2,3]. As per literature the incidence of rotator cuff calcific tendinitis ranges from $6.8 \%$ to $54 \%$ [4]. Etiology is still disputed and is considered idiopathic. However, various theories have been proposed over the years including ischemia/ hypoxia, metabolic disturbances, endocrine disturbances involving abnormal activities of the thyroid gland and diabetes, localized pressure/microtrauma, fibrocartilaginous transformation of the degenerated tendon and genetic predisposition. Of these degenerative and multiphasic ones are the most popular ones Many authors consider the origin to be multifactorial resulting from a combination of both extrinsic and intrinsic factors [2,5]. Treatment is usually conservative and intervention like Barbotage and surgery is required only occasionally. We present a case report of a patient who presented with calcific deposits in an unusual location that was successfully treated with a combination of conservative therapy and barbotage.

\section{Case Report}

A 45 year old female patient presented with pain in her right shoulder which progressed over a period of 2-3 days to severe pain. The pain was severe enough to restrict all the movements of the shoulder and interfere with her sleep. The patient was an average healthy female with no known medical illnesses and she was not on any drugs. However, she was undergoing perimenopausal dysfunctional uttering bleeding at the time of presentation. For this she was not on any medication. Her vitamin D levels were $30 \mathrm{ng} / \mathrm{ml}$. She had been having dull aching pain especially in abduction and external rotation for the past 04 years. Radiographs performed during these 04 years revealed two well defined calcific deposits superior to her glenoid and deep to her acromioclavicular joint measuring $11 \times 7.2 \times 5.4 \mathrm{~mm}$ in size (together). While individually they measured $5 \times 3.1 \times 2.6$ and $6.1 \times 3.6 \times 2.4 \mathrm{~mm}$ each) (Figure 1). Of these one became more fluffy and ill-defined, while the other retained its original shape (Figure 2). An MRI scan performed after the first radiograph revealed two well-defined calcific deposits positioned one over the other in the supraglenoid region deep to the supraspinatus muscle belly. These together measured $15 \mathrm{x} 11.5 \mathrm{x} 5 \mathrm{~mm}$. The rotator cuff and the glenohumeral joint appeared normal (Figure 3). During the present episode of severe pain radiograph of the shoulder AP oblique view was performed which revealed only one calcific deposit which appeared fluffy in outline (Figure 4). An MRI scan performed at this 
time revealed inflammatory changes surrounding the calcific deposit which measured 20x 12.4x 5.2mm, an erosion of the superior glenoid and significant joint effusion (Figure 5). In view of the severe pain and the appearance of calcific deposit the patient opted to undergo Barbotage. Because of the location of the deposits these were not visible on ultrasonography. Hence, the procedure was performed under fluoroscopy guidance from the posterior aspect. In view of the large volume of the deposit it was targeted directly. After injection of local anaesthetic, a $22 \mathrm{G}$ needle attached to a syringe of normal saline was inserted and aspiration of calcium was performed. Following this $1 \mathrm{ml}$ of Traimcinolone acetate and $1 \mathrm{ml}$ of $0.5 \%$ Bupivacaine was injected into the joint space (Figure 6). There was significant relief in pain following the procedure and there was no recurrence of pain on the following day or after that. Pathological examination of the aspirated material revealed non-bi-referingent crystals. An MRI scan performed immediately after the procedure (i,e 05 days after the pre-procedure MRI) revealed significant residual calcific deposit (measuring $14 \times 3.4 \times 5.3 \mathrm{~mm}$ ) (Figure 7). It was more heterogenous in intensity pattern. The patient opted for further conservative treatment as there was no pain. An MRI scan performed 02 months later revealed only a residual erosion of the superior portion of the Glenoid and complete resolution of the calcific deposit (Figure 8). Patient continued to remain symptom free. A follow-up MRI performed 02 year later revealed complete resolution of the calcific deposit and the erosion (Figure 9). There has been no recurrence on a further follow-up of 01 more years.

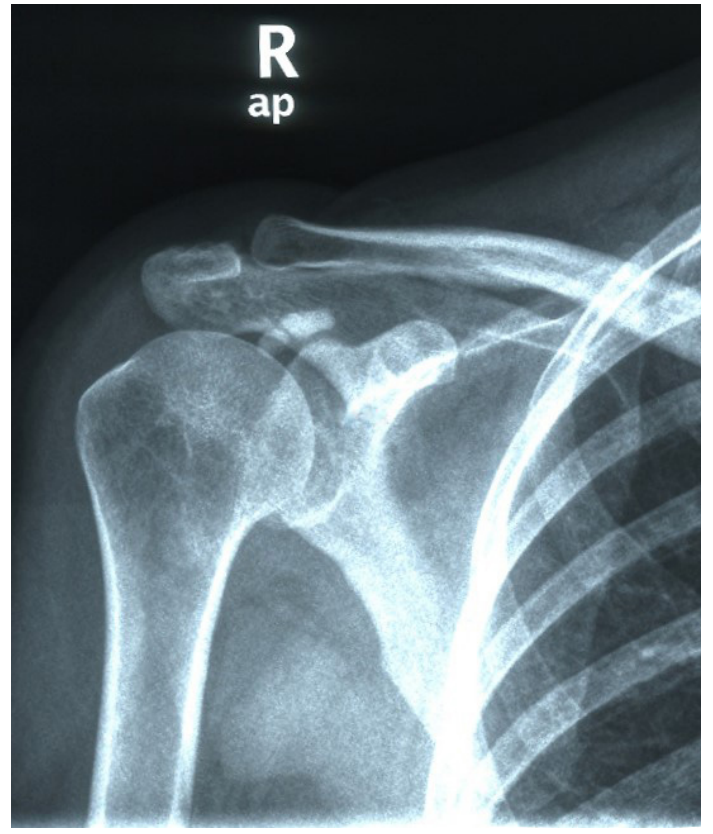

Figure 1: Antero-posterior radiograph of right shoulder taken in July 2012, reveals two well defined calcific deposits superior to her glenoid and deep to her acromio-clavicular joint measuring approximately $1 \mathrm{~cm}$ in size

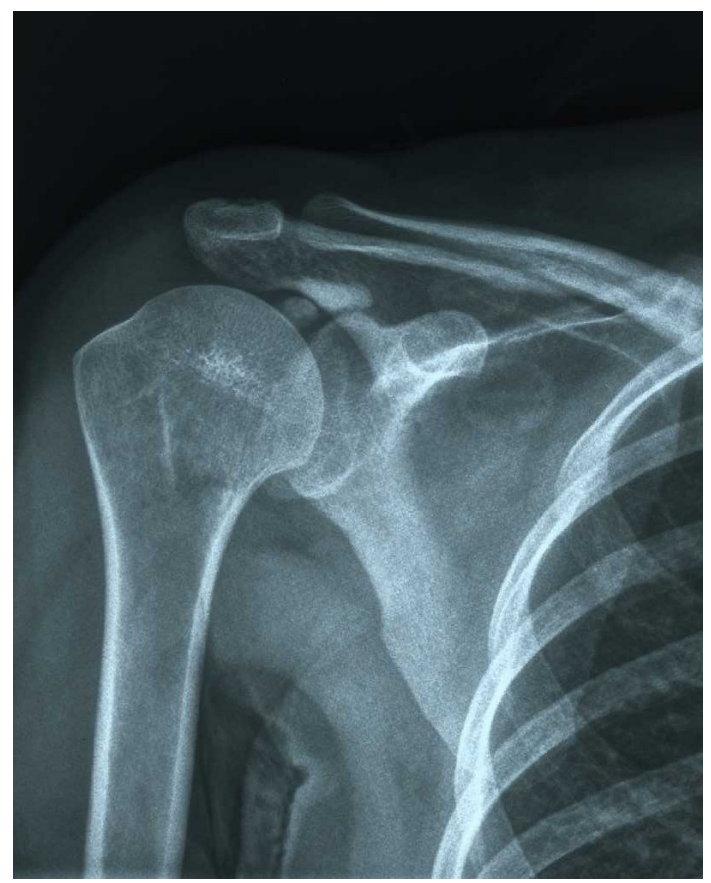

Figure 2: Anteroposterior radiograph of the right shoulder taken in October 2013, reveals that one of the deposit has become more fluffy while the other has retained its shape 


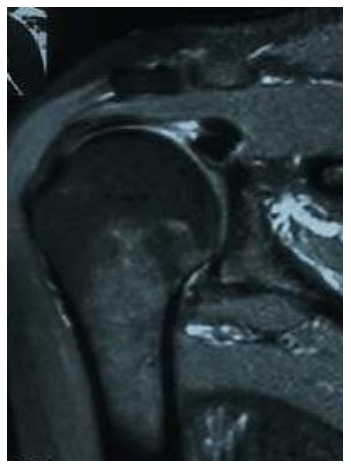

Figure 3: Fat suppressed proton density weighted MRI image reveals two well-defined calcific deposits positioned one over the other in the supra-glenoid region deep to the supraspinatus muscle belly. The rotator cuff and the gleno-humeral joint appeared normal

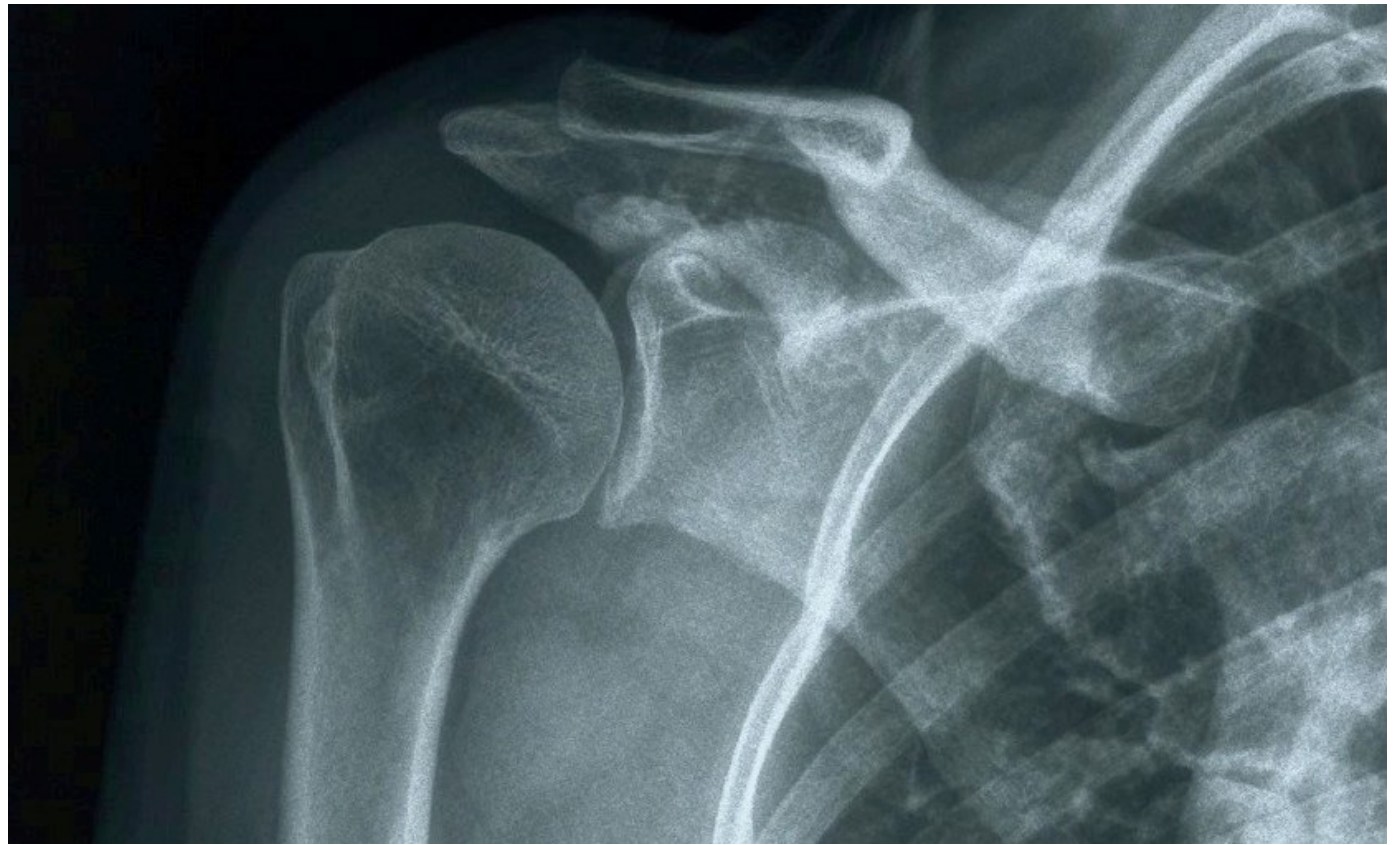

Figure 4: AP oblique view of the right shoulder joint taken in August 2015, reveals only one calcific deposit which appeared fluffy in outline
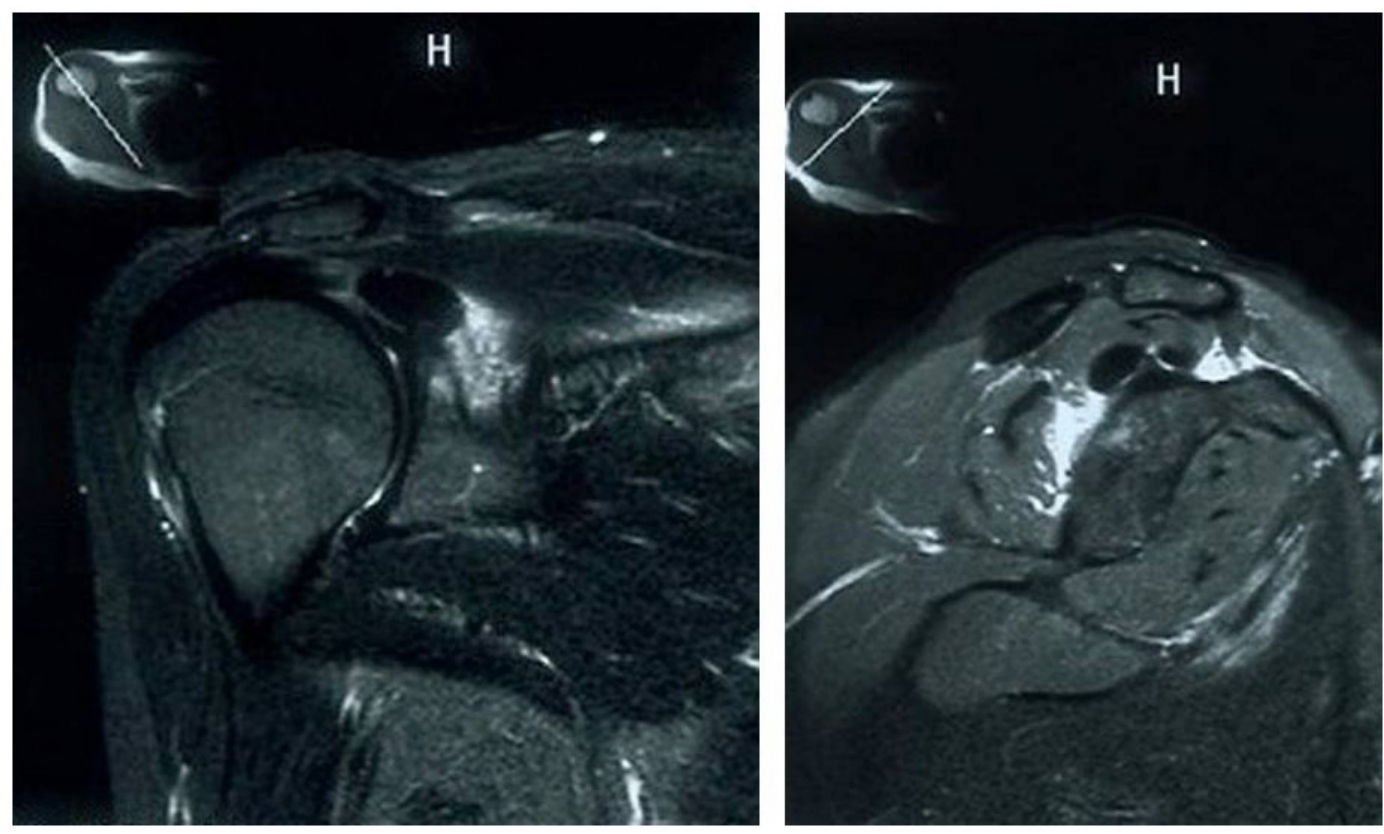

Figure 5: Coronal and sagittal fat suppressed proton density weighted MRI images reveal inflammatory changes surrounding the calcific deposit, an erosion of the superior glenoid and significant joint effusion 


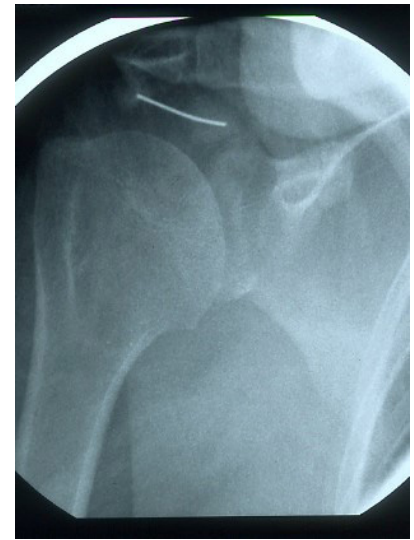

Figure 6: Radiograph of the right shoulder obtained during fluoroscopy with the needle in situ targeting the calcific deposit

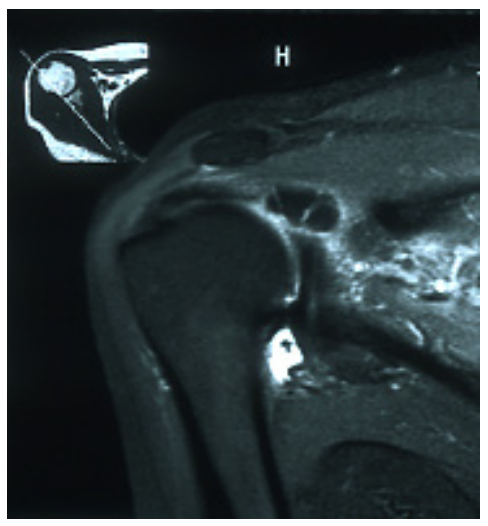

Figure 7: Coronal fat suppressed proton density weighted MRI image performed immediately after the procedure on the next day revealed significant residual calcific deposit

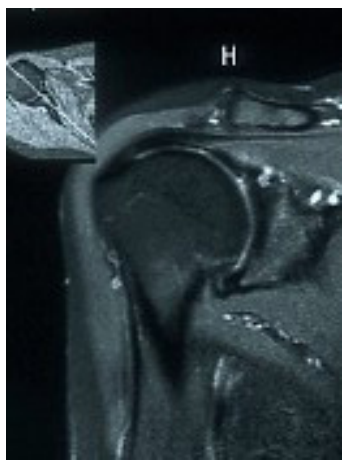

Figure 8: Coronal fat suppressed proton density weighted MRI image performed 02 months later revealed only a residual erosion of the superior portion of the Glenoid and complete resolution of the calcific deposit

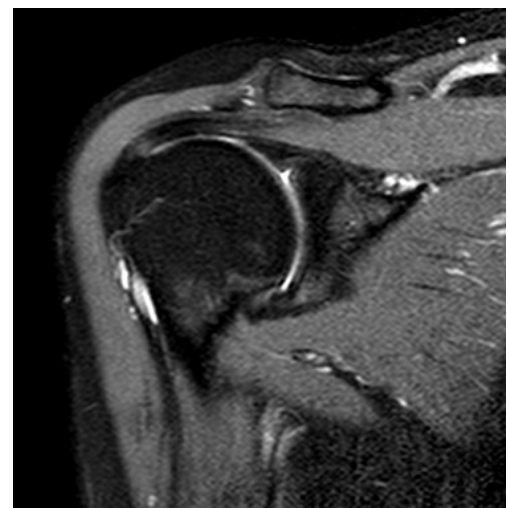

Figure 9: A follow-up MRI performed 02 year later reveals complete resolution of the calcific deposit and the erosion 


\section{Discussion}

Hydroxyapatite deposition disease can either present as calcific tendinitis, other periarticular hydroxyapatite deposition, and hydroxyapatite induced arthritis [1]. Radiographs can demonstrate these deposits in upto 7.5-20\% of asymptomatic adults [6]. Clavert et al. found the prevalence of 7.3\% in their study of 1276 asymptomatic shoulders [7]. Based on the progression of the disease four stages of this disease have been described; pre-calcific (stage 1) which is usually asymptomatic and is characterized by fibrocartilaginous transformation of the tendon fibers; formative or Stage 2 has well-formed calcifications and is associated with some symptoms like low grade pain increasing at night; resorptive or Stage 3 has increased vasculature and calcium deposits which are usually removed by phagocytes; and post-calcific (Stage 4), in which self- healing and repair of the tendon fibers occurs [2]. There is another classification described by Gärtner which describes its natural course and has been associated with prognosis. The Type I calcification have a sharp border and a dense structure, the Type III calcification has vague border and is more or less transparent [8]. There was gradual progression from Type I to Type III in the patient reported. De Witte et al. found barbotage to be superior in cases of Type II and Type III calcification rather than Gärtner Type I calcification [9]. These features of calcific deposits i.e number, size, appearance and location of calcification have prognostic significance according to some authors, but it has been disputed by other [4]. One of the focuses of calcifications in the case described was Type I and it disappeared spontaneously while the other progressed to Type III and Barbotage was performed for this patient.

Out of all the cases of crystal deposition disease only 50\% become symptomatic [6]. Treatment is generally conservative with immobilization with sling, non-steroidal anti-inflammatory drugs, and physical therapy. It is successful in upto 90\% of patients [9]. However, in case of persisting and severe symptoms invasive treatments like corticosteroid injections, barbotage, extracorporeal shock wave therapy (ESWT), acetic acid iontophoresis or surgery are applied [10]. Surgery is most effective treatment available; however it is associated with its own complications, requires rehabilitation and is associated with greater health costs. Shock wave treatment has also been found useful for treating calcific tendonitis; however, it is painful [10]. Many different techniques have been described by various authors. Some authors use two needles, while the others use only a single needle. Some aspirate, while the others do not. Jelsingh et al. described a simple technique to restore the patency of needle by using a $25 \mathrm{G}$ needle to restore the patency of an obstructed 20G needle [11]. Cura et al. found that the outcome of the patients in whom calcium was aspirated was not significantly different from that of patients in whom this was not the case. Hence, they hypothesized that even if the calcium was not extracted, the injection somehow excited an inflammatory process that removed the calcification. They also found that half their patients experienced recurrence of symptoms at approximately 15 weeks after treatment which lasted for about 6 weeks. They also observed that frequently there was a temporary worsening of symptoms that lasted for approximately 48 hours [10]. In the case presented there was significant residual deposit which disappeared completely subsequently indicating that complete aspiration of the caicific deposit is not necessary. Also there was no worsening or recurrence of symptoms at 02 years follow-up.

\section{Conclusion}

Hydroxyapatite deposition disease is a self-limiting disease. It can however, present with very disabling symptoms. Treatment options are still evolving. To date Barbotage is the most popular treatment available. This technique has its inherent problems which are continuously being resolved by various authors, as is evident in the literature. Hence, it is important to approach each case individually. The treatment decision should involve the location of the calcific deposit, stage of calcification, volume of the deposit and the symptoms of the patient. The right management decision can help resolve this condition completely.

The authors certify that they have obtained all appropriate patient consent forms. In the form the patient(s) has/have given his/ her/their consent for his/her/their images and other clinical information to be reported in the journal. The patients understand that their names and initials will not be published and due efforts will be made to conceal their identity, but anonymity cannot be guaranteed.

\section{References}

1. Garcia GM, McCord GC, Kumar R (2003) Hydroxyapatite crystal deposition disease. Semin Musculoskelet Radiol 7: 187-93.

2. Della Valle V, Bassi EM, Calliada F (2015) Migration of calcium deposits into subacromial-subdeltoid bursa and into humeral head as a rare complication of calcifying tendinitis: sonography and imaging. J Ultrasound 18: 259-63.

3. Chen SK, Chou PH, Lue YJ, Lu YM (2008) Treatment for frozen shoulder combined with calcific tendinitis of the supraspinatus. Kaohsiung J Med Sci 24: 78-84. 4. de Witte PB, van Adrichem RA, Selten JW, Nagels J, Reijnierse M, et al. (2016) Radiological and clinical predictors of long-term outcome in rotator cuff calcific tendinitis. Eur Radiol 26: 3401-11.

5. Jelsing EJ, Maida E, Smith J (2013) A simple technique to restore needle patency during percutaneous lavage and aspiration of calcific rotator cuff tendinopathy. PM R 5: 242-4.

6. Klontzas ME, Vassalou EE, Zibis AH, Karantanas AH (2016) Hydroxyapatite deposition disease around the hip: outcomes of CT-guided treatment. Diagn Interv Radiol 22: 466-70.

7. Clavert P, Sirveaux F (2008) Shoulder calcifying tendinitis. Revue de chirurgie orthopedique et reparatrice de l'appareil moteur 94: $336-55$.

8. Gartner J, Simons B (1990) Analysis of calcific deposits in calcifying tendinitis. Clin Orthop Relat Res 254: 111-20.

9. de Witte PB, Selten JW, Navas A, Nagels J, Visser CP, et al. (2013) Calcific tendinitis of the rotator cuff: a randomized controlled trial of ultrasound-guided needling and lavage versus subacromial corticosteroids. Am J Sports Med 41: 1665-73. 
10. del Cura JL, Torre I, Zabala R, Legorburu A (2007) Sonographically guided percutaneous needle lavage in calcific tendinitis of the shoulder: short- and longterm results. AJR Am J Roentgenol 189: W128-34.

11. Jelsing EJ, Maida E, Smith J (2013) A simple technique to restore needle patency during percutaneous lavage and aspiration of calcific rotator cuff tendinopathy. PM R 5: 242-4. 